\title{
Existence, optimality and dynamics of equilibria with endogenous time preference
}

\author{
Selman Erol ${ }^{\mathrm{a}}$, Cuong Le Van ${ }^{\mathrm{b}, \mathrm{c}, \mathrm{d}}$, Cagri Saglam ${ }^{\mathrm{a}, *}$ \\ a Department of Economics, Bilkent University, Turkey \\ ${ }^{\mathrm{b}}$ CNRS, Paris School of Economics, France \\ ${ }^{\mathrm{c}}$ Exeter University Department of Economics, United Kingdom \\ d DEPOCEN, Viet Nam
}

\section{A R T I C L E I N F O}

\section{Article history:}

Received 25 July 2009

Received in revised form

20 December 2010

Accepted 26 December 2010

Available online 19 February 2011

\section{JEL classification:}

C61

D90

041

Keywords:

Endogenous time preference

Optimal growth

Competitive equilibrium

Multiple steady-states

\begin{abstract}
A B S T R A C T
This paper studies the dynamic implications of the endogenous rate of time preference depending on the stock of capital, in a one-sector growth model. The planner's problem is presented and the optimal paths are characterized. We prove that there exists a critical value of initial stock, in the vicinity of which, small differences lead to permanent differences in the optimal path. Indeed, we show that a development trap can arise even under a strictly convex technology. In contrast with the early contributions that consider recursive preferences, the critical stock is not an unstable steady state so that if an economy starts at this stock, an indeterminacy will emerge. We also show that even under a convex-concave technology, the optimal path can exhibit global convergence to a unique stationary point. The multipliers system associated with an optimal path is proven to be the supporting price system of a competitive equilibrium under externality and detailed results concerning the properties of optimal (equilibrium) paths are provided. We show that the model exhibits globally monotone capital sequences yielding a richer set of potential dynamics than the classic model with exogenous discounting.
\end{abstract}

(C) 2011 Elsevier B.V. All rights reserved.

\section{Introduction}

The optimal capital accumulation models have been at the core of the theory of economic growth and dynamics. Based on the dynamic consumption and saving decisions of the economic agents driven by intertemporal utility trade-offs between current and future consumption, the key components of these models turn out to be the rate of time preference and the technology. The classical optimal growth models and much of the subsequent literature on growth focus on the convex structures of the technology and preferences that guarantee the monotonical convergence of the sequence of optimal stocks towards a unique steady state. Such a structure, however, imply that the model cannot be used to understand the development patterns that differ considerably among countries in the long run (see Quah, 1996; Barro, 1997; Barro and Sala-i-Martin, 1991).

\footnotetext{
* Corresponding author. Tel.: +90 312 2901598; fax: +90 3122665140.

E-mail addresses: selman@bilkent.edu.tr(S. Erol), levan@univ-paris1.fr (C. Le Van), csaglam@bilkent.edu.tr (C. Saglam).
}

To account for these non-convergent growth paths, a variety of one-sector optimal growth models that incorporate some degree of market imperfections based on technological external effects and increasing returns have been presented. Within a model of capital accumulation with convex-concave technology, Dechert and Nishimura (1983), Mitra and Ray (1984) have characterized optimal paths and prove the existence of threshold dynamics that generate development or poverty traps (see Azariadis and Stachurski, 2005, for a recent survey). In these models, economies with low initial capital stocks or incomes converge to a steady state with low per capita income, while economies with high initial capital stocks converge to a steady state with high per capita income. Indeed, the introduction of increasing returns also makes it possible for private returns to the accumulation of capital stock to be complementary with the aggregate stock leading to indeterminacies or continuum of equilibria (e.g., Benhabib and Perli, 1994; Benhabib and Farmer, 1996; see Nishimura and Venditti, 2006 for extensive bibliography).

A general tendency in these studies with multiple steady states, indeterminacy or continuum of equilibria is that they are mostly devoted to the analysis of the technology component leaving the time preference essentially unaltered with an exogenously fixed 
geometric discounting. In this paper, yet the other mechanism, the endogeneity of time preference will be put forward to explain theoretically why the differences in per capita output levels among countries persist in the long run.

Considering the preferences of the agents to be recursive, the early contributions on the theory of endogenous time preference postulate that an agent's discount rate depends on the level of present and future consumption (e.g., Uzawa, 1968; Lucas and Stokey, 1984; Epstein, 1987; Obstfeld, 1990). These models nearly exclusively assume an increasing marginal impatience so that the agents get more impatient as they grow richer. Such a specification ensures stable optimal capital sequences that converge to a unique steady state independent of the initial conditions. However, recent work both theoretically (e.g., Becker and Mulligan, 1997; Stern, 2006) and empirically (e.g., Lawrance, 1991; Samwick, 1998; Frederick et al., 2002) propose that the agents get more patient as they grow richer and impose that the discount rate depends closely upon the stock of wealth (see Hamada and Takeda, 2009, for a recent survey). This is in parallel to the idea that the stock of wealth is a key to reach better health services and better insurance markets so that the more wealthier the agent gets, the more patient it becomes. By considering discount factor as a function of consumption, Mantel (1998) studies the impact of decreasing rate of time preference on optimal growth path of an economy with a primary focus on the monotonicity properties of optimal consumption and investment. Das (2003) derives a set of sufficient conditions for stability and uniqueness in the same context in a continuous time optimal growth model. Stern (2006), along the lines of Becker and Mulligan (1997), let individuals spend resources to increase the appreciation of the future. He provides numerical examples of multiple steady states and a conditionally sustained growth path.

In this paper we adapt the classic optimal growth model to include an endogenous rate of time preference depending on the stock of capital and analyze the implications on the equilibrium dynamics. To do so, the planner's problem is first presented and the optimal paths are characterized. We show that a development trap can arise even under a strictly convex technology. In other words, we prove that there exists a critical value of initial stock, in the vicinity of which, small differences lead to permanent differences in the optimal path. In contrast with the early contributions by Kurz (1968), who assumes capital dependent preferences, by Beals and Koopmans (1969), Iwai (1972), who assume recursive preferences, and more recently, by Stern (2006), who assumes endogenous time preference depending on future oriented resources, we noted that the critical stock is definitely not an unstable steady state so that if an economy starts at this stock, an indeterminacy will emerge. On the other hand, under a similar condition to that of Dechert and Nishimura (1983), we also show that even under a convex-concave technology, the optimal path can exhibit global convergence to a unique stationary point. Later, the multipliers system associated with an optimal path is proven to be the supporting price system of a competitive equilibrium under externality and detailed results concerning the properties of optimal (equilibrium) paths are provided. We show that the model exhibits globally monotone capital sequences yielding a richer set of potential dynamics than the classic model with exogenous discounting.

The key reason of our results is that we consider that the rate of time preference decreases with the stock of wealth in contrast with exogenously fixed discount factor assumed in standard models. In particular, the discount factor attributed to the utility of consumption at period $t$ increases with the level of capital stock available for production at period $t$. Accordingly, the lower the stock of capital, the higher the sacrifice of postponing present consumption in exchange for future consumption. In line with Becker and Mulligan (1997), Stern (2006), an immediate implication of this assumption is that as the agent grows wealthier, the level of investment in capital that will be appreciated in the future increases and therefore the agent will become more patient. Indeed, recent empirical studies using experimental data also support the validity of this hypothesis of wealth causes patience (e.g., Harrison et al., 2002; Ikeda et al., 2005).

Incorporating such an hypothesis, an important aspect of our model is that while allowing variation in the rate of time preference, at the same time it maintains time consistency. A remarkable feature of our analysis is that our results do not rely on particular parameterization of the exogenous functions involved in the model, rather, it provides a more flexible framework in regards to the discounting of time, keeps the model analytically tractable and uses only general and plausible qualitative properties.

The rest of the paper is organized as follows. Section 2 describes the model and provides the dynamic properties of optimal paths. Section 3 presents the existence of a competitive equilibrium with externality and studies the equilibrium dynamics. Finally, Section 4 concludes.

\section{Model}

The model differs from the classic optimal growth model by the assumptions on discounting. Rather than assuming that the level of discount on future utility is an exogenous parameter, we assume that it is endogenous depending on the path of capital stock. Formally, the model is stated as follows:

$\max _{\left\{c_{t}, x_{t+1}\right\}_{t=0}^{\infty}} \sum_{t=0}^{\infty}\left(\prod_{s=1}^{t} \beta\left(x_{s}\right)\right) u\left(c_{t}\right)$

subject to

$\forall t, c_{t}+x_{t+1} \leq f\left(x_{t}\right)$

$\forall t, c_{t} \geq 0, x_{t} \geq 0$,

$x_{0} \geq 0$,given,

We make the following assumptions regarding the properties of the discount, utility and the production functions.

Assumption 1. $\beta: \mathbb{R}_{+} \rightarrow \mathbb{R}_{++}$is continuous, differentiable, strictly increasing and satisfies $\sup _{x>0} \beta(x)=\beta_{m}<1, \quad \sup _{x>0} \beta^{\prime}$ $(x)<+\infty$.

Assumption 2. $u: \mathbb{R}_{+} \rightarrow \mathbb{R}_{+}$is continuous, twice continuously differentiable and satisfies $u(0)=0$. Moreover, it is strictly increasing, strictly concave and $u^{\prime}(0)=+\infty$ (Inada condition).

Assumption 3. $f: \mathbb{R}_{+} \rightarrow \mathbb{R}_{+}$is continuous, twice continuously differentiable and satisfies $f(0)=0$. Moreover, it is strictly increasing and $\lim _{x \rightarrow+\infty} f^{\prime}(x)<1$.

For any initial condition $x_{0} \geq 0$, when $\mathbf{x}=\left(x_{0}, x_{1}, x_{2}, \ldots\right)$ is such that $0 \leq x_{t+1} \leq f\left(x_{t}\right)$ for all $t$, we say it is feasible from $x_{0}$ and the class of all feasible accumulation paths is denoted by $\Pi\left(x_{0}\right)$. It may be easily verified that if $x_{0}<x_{0}^{\prime}$, then $\Pi\left(x_{0}\right) \subset \Pi\left(x_{0}^{\prime}\right)$. A consumption sequence $\mathbf{c}=\left(c_{0}, c_{1}, \ldots\right)$ is feasible from $x_{0} \geq 0$ when there exists $\mathbf{x} \in \Pi\left(x_{0}\right)$ with $0 \leq c_{t} \leq f\left(x_{t}\right)-x_{t+1}$.

Noting that the constraints will be binding at the optimum as the utility and the discount functions are strictly increasing, we introduce the function $U$ defined on the set of feasible sequences as

$U(\mathbf{x})=\sum_{t=0}^{\infty}\left(\prod_{s=1}^{t} \beta\left(x_{s}\right)\right) u\left(f\left(x_{t}\right)-x_{t+1}\right)$.

The preliminary results are summarized in the following Lemma which has a standard proof using Tychonov theorem (see Le Van and Dana, 2003; Duran and Le Van, 2003; Stokey et al., 1989). 
Lemma 1. Let $\bar{x}$ be the greatest point $x \geq 0$ such that $f(x)=x$. Then,

(a) For any $\mathbf{x} \in \Pi\left(x_{0}\right)$, we have $x_{t} \leq A\left(x_{0}\right)$ for all $t$, where $A\left(x_{0}\right)=$ $\max \left\{x_{0}, \bar{x}\right\}$.

(b) $\Pi\left(x_{0}\right)$ is compact in the product topology defined on the space of sequences $\mathbf{x}$.

(c) $U$ is well defined and it is continuous over $\Pi\left(x_{0}\right)$ with respect to the product topology.

It is now clear that the initial optimal growth model is equivalent to:

$\max \left\{U(\mathbf{x}) \mid \mathbf{x} \in \Pi\left(x_{0}\right)\right\}$.

\subsection{Existence of optimal paths}

The existence of an optimal path follows from the fact that $\Pi\left(x_{0}\right)$ is compact for the product topology defined on the space of sequences $\mathbf{x}$ and $U$ is continuous for this product topology. The positivity of optimal consumption and capital stock paths follow from the Inada condition.

\section{Proposition 1.}

(i) There exists an optimal path $\mathbf{x}$. The associated optimal consumption path, $\mathbf{c}$ is given by

$c_{t}=f\left(x_{t}\right)-x_{t+1}, \forall t$

(ii) If $x_{0}>0$, every solution $(\mathbf{x}, \mathbf{c})$ to the optimal growth model satisfies

$c_{t}>0, x_{t}>0, \forall t$

Proof. It is easy.

\subsection{Value function, Bellman equation, optimal policy}

In order to characterize the behavior of the optimal paths, we will proceed by defining the value function and analyzing the properties of the optimal policy correspondence. The value function $V$ is defined by:

$$
\begin{aligned}
& \forall x_{0} \geq 0, V\left(x_{0}\right)=\max _{\left\{x_{t+1}\right\}_{t=0}^{\infty}}\left\{\sum_{t=0}^{\infty}\left(\prod_{s=1}^{t} \beta\left(x_{s}\right)\right) u\left(f\left(x_{t}\right)-x_{t+1}\right)\right. \\
& \left.\forall t, 0 \leq x_{t+1} \leq f\left(x_{t}\right), x_{0} \geq 0 \text {,given }\right\} .
\end{aligned}
$$

The bounds on discounting together with the existence of maximum sustainable capital stock guarantee a finite value function. Under the Assumption (1) and the Assumption (2), one can immediately show that the value function is non-negative, strictly increasing and continuous (see Stokey et al., 1989). Given these, Bellman equation follows.

\section{Proposition 2.}

(i) $V(0)=0$, and $V\left(x_{0}\right)>0$ if $x_{0}>0$. If $\mathbf{x}$ is an optimal path, then

$$
V\left(x_{0}\right)=\sum_{t=0}^{\infty}\left(\prod_{s=1}^{t} \beta\left(x_{s}\right)\right) u\left(f\left(x_{t}\right)-x_{t+1}\right) .
$$

(ii) V is strictly increasing.

(iii) $V$ is continuous.

\section{Proposition 3.}

(i) V satisfies the following Bellman equation:

$$
\forall x_{0} \geq 0, V\left(x_{0}\right)=\max \left\{u\left(f\left(x_{0}\right)-x\right)+\beta(x) V(x) \mid 0 \leq x \leq f\left(x_{0}\right)\right\} .
$$

(ii) A sequence $\mathbf{x} \in \Pi\left(x_{0}\right)$ is an optimal solution if and only if it satisfies:

$$
\forall t, V\left(x_{t}\right)=u\left(f\left(x_{t}\right)-x_{t+1}\right)+\beta\left(x_{t+1}\right) V\left(x_{t+1}\right) .
$$

Proof. See Le Van and Dana (2003).

The optimal policy correspondence, $\mu: \mathbb{R}_{+} \rightarrow \mathbb{R}_{+}$is defined as follows:

$\mu\left(x_{0}\right)=\arg \max \left\{u\left(f\left(x_{0}\right)-y\right)+\beta(y) V(y) \mid y \in\left[0, f\left(x_{0}\right)\right]\right\}$.

It is important to note that although the utility function is strictly concave, the solution may not be unique as the multiplication of discount function destroys the concave structure needed for uniqueness. The non-emptiness and the closedness of the optimal correspondence and its equivalence with the optimal path follow easily from the continuity of the value function by a standard application of the theorem of the maximum.

\section{Proposition 4.}

(i) $\mu(0)=\{0\}$.

(ii) If $x_{0}>0$ and $x_{1} \in \mu\left(x_{0}\right)$, then $0<x_{1}<f\left(x_{0}\right)$.

(iii) $\mu$ is closed and hence upper semi-continuous.

(iv) A sequence $\mathbf{x} \in \Pi\left(x_{0}\right)$ is optimal if and only if $x_{t+1} \in \mu\left(x_{t}\right), \forall t$.

(v) The optimal correspondence $\mu$ is increasing so that if $x_{0}<x_{0}^{\prime}$, $x_{1} \in \mu\left(x_{0}\right)$ and $x_{1}^{\prime} \in \mu\left(x_{0}^{\prime}\right)$ then $x_{1}<x_{1}^{\prime}$.

Proof. (ii) Follows easily from (2). (iii) Follows from the Theorem of Maximum. (iv) Follows from (5). (v) See Dechert and Nishimura (1983), or Amir et al. (1991).

The increasingness of $\mu$ is crucial for the convergence of optimal paths, hence for the analysis of the long-run dynamics. In Stern (2006), assuming an endogenous time preference depending on the future-oriented resources, the increasingness of $\mu$ has been proven by using the strict concavity of $\beta$. Such a restriction on the curvature of the discount function is not necessary in our setup. Moreover, we have also proven that the optimal correspondence, $\mu$ is not only closed but also upper semi-continuous.

With the positivity of the optimal consumption and the stock of capital, Euler equation easily follows.

Proposition 5. When $x_{0}>0$, any solution $\mathbf{x} \in \Pi\left(x_{0}\right)$ satisfies the Euler equation:

$$
\begin{aligned}
& \forall t, u^{\prime}\left(f\left(x_{t}\right)-x_{t+1}\right)=\beta\left(x_{t+1}\right) u^{\prime}\left(f\left(x_{t+1}\right)-x_{t+2}\right) f^{\prime}\left(x_{t+1}\right) \\
& \quad+\beta^{\prime}\left(x_{t+1}\right) V\left(x_{t+1}\right)
\end{aligned}
$$

Proof. Recall that $0<x_{t+1}<f\left(x_{t}\right), \forall t$. Take any $n$, and consider the path of capital $\mathbf{x}^{y}$ defined as follows: $x_{t}^{y}=x_{t}, \forall t \neq n+1$ and $x_{n+1}^{y}=$ $y \in Y$, where $Y=\left(f^{-1}\left(x_{n+2}\right), f\left(x_{n}\right)\right)$. Note that it is a well defined open interval including $x_{n+1}$ because $x_{t+1}<f\left(x_{t}\right)$ and $x_{t+2}<f\left(x_{t+1}\right)$. By choosing $y \in Y$, we guarantee that $y<f\left(x_{t}\right)$ and $x_{t+2}<f(y)$ so that $\mathbf{x}^{y} \in \Pi\left(x_{0}\right)$. From the optimality of $\mathbf{x}$ we have $U(\mathbf{x}) \geq U\left(\mathbf{x}^{y}\right), \forall y \in Y$. Let $\Psi(y)=U\left(\mathbf{x}^{y}\right)$. Then $\Psi(y)$ is maximized at zero in $Y$, which suggests that $\Psi^{\prime}(0)=0$. With some algebra, this ends the proof.

In a standard optimal growth model with geometric discounting and the usual concavity assumptions on preferences and technology, the optimal policy correspondence, $\mu$ is single valued and 
the properties of the optimal path is easily found by using the first order conditions together with envelope theorem by differentiating the value function. However, in our model, the objective function includes multiplication of a discount function. This generally destroys the usual concavity argument which is used in the proof of the differentiability of value function and the uniqueness of the optimal paths (see Benveniste and Scheinkman, 1979; Araujo, 1991).

To this end, the next proposition shows that the value function is differentiable almost everywhere and there exists a unique optimal path from almost everywhere without any assumption on the curvature of $\beta$.

\section{Proposition 6.}

(i) Left derivative of $V$ exists at every $x_{0}>0$ and $V_{-}^{\prime}\left(x_{0}\right)=u^{\prime}\left(f\left(x_{0}\right)-\right.$ $\left.\theta\left(x_{0}\right)\right) f^{\prime}\left(x_{0}\right)$, where $\theta\left(x_{0}\right)=\min \mu\left(x_{0}\right)$.

(ii) Right derivative of $V$ exists at every $x_{0}>0$ and $V_{+}^{\prime}\left(x_{0}\right)=u^{\prime}\left(f\left(x_{0}\right)-\right.$ $\left.\Theta\left(x_{0}\right)\right) f^{\prime}\left(x_{0}\right)$, where $\Theta\left(x_{0}\right)=\max \mu\left(x_{0}\right)$.

Proof. See Askri and Le Van (1998); Le Van and Dana (2003) for the proofs based on the Clarke generalized gradients and the standard optimal growth tools, respectively.

Now we are able to see the relation between differentiability of the value function and the uniqueness of the optimal path. Notice that, given $x_{0}$, if optimal $x_{1}$ is unique so that $\mu\left(x_{0}\right)$ is singlevalued and $\theta\left(x_{0}\right)=\Theta\left(x_{0}\right)=x_{1}$, from the above proposition, we see that $V_{-}^{\prime}\left(x_{0}\right)=V_{+}^{\prime}\left(x_{0}\right)$, hence $V$ is differentiable at $x_{0}$, and vice versa. This analysis can be generalized to any period $t$ other than zero. These allow us to claim that the optimal correspondence $\mu$ is single valued and differentiable almost everywhere.

\section{Proposition 7.}

(i) If $\mathbf{x}$ is an optimal path from $x_{0}$, then $V$ is differentiable at any $x_{t}$, $t \geq 1$. If $\mathbf{x}$ is an optimal path from $x_{0}$, there exists a unique optimal path from $x_{t}$ for any $t \geq 1$.

(ii) $V$ is differentiable at $x_{0}>0$ if and only if there exists a unique optimal path from $x_{0}$.

(iii) $V$ is differentiable almost everywhere, i.e., the optimal path is unique for almost every $x_{0}>0$.

(iv) $\mu$ is differentiable almost everywhere.

Proof. See Le Van and Dana (2003).

We have proved that the optimal correspondence $\mu$ is single valued and differentiable almost everywhere. In addition to this, we have also shown that there exists a unique optimal path from almost any initial capital stock. These results will prove to be crucial in analyzing the dynamic properties of the optimal paths.

\subsection{Dynamic properties of the optimal paths}

A point $x^{s}$ is an optimal steady state if $x^{s}=\mu\left(x^{s}\right)$, so that the stationary sequence $\mathbf{x}^{s}=\left(x^{s}, x^{s}, \ldots, x^{s}, \ldots\right)$ solves the problem: $\max \left\{U\left(\mathbf{x}^{s}\right): \mathbf{x}^{s} \in \Pi\left(x^{s}\right)\right\}$. If $x^{s}$ is different from zero, then the associated optimal steady state consumption must be strictly positive from Inada condition. Hence, from Euler equation (6), this steady state will solve:

$u^{\prime}(f(x)-x)=\beta(x) u^{\prime}(f(x)-x) f^{\prime}(x)+\beta^{\prime}(x) V(x)$.

By Proposition 3, we know that the stationary plan every period equal to $x^{S}$ is optimal from $x^{S}$ if and only if it satisfies

$V\left(x^{s}\right)=u\left(f\left(x^{s}\right)-x^{s}\right)+\beta\left(x^{s}\right) V\left(x^{s}\right)$.
Following from (7) and (8), this steady state will satisfy:

$u(f(x)-x)=\frac{[1-\beta(x)]\left[1-\beta(x) f^{\prime}(x)\right]}{\beta^{\prime}(x)} u^{\prime}(f(x)-x)$.

We will now prove that the endogenous rate of time preference preserves the monotonicity of the optimal paths and provide the condition under which the convergence to an optimal steady state is guaranteed.

Proposition 8. The optimal path $\mathbf{x}$ from $x_{0}$ is monotonic.

Proof. Since $\mu$ is increasing, if $x_{0}>x_{1}$, we have $x_{1}>x_{2}$. By induction, $x_{t}>x_{t+1}, \forall t$. If $x_{1}>x_{0}$, using the same argument yields $x_{t+1}>x_{t}$, $\forall t$. Now if $x_{1}=x_{0}$, then $x_{0} \in \mu\left(x_{0}\right)$. Recall that the optimal path is unique after $t=1$. Since $x_{0} \in \mu\left(x_{0}\right), x_{t}=x_{0}, \forall t$.

It is important to note that the monotonicity of the optimal paths has been proved without any assumption on the curvature of neither the production nor the discount function. It is already well known that in multi-sector nonclassical optimal growth models, one can easily refer to lattice programming and Topkis theorem in order to prove that the optimal paths are monotonic if the planner's criterion function is supermodular (see Amir et al., 1991). However, following this approach in a model with time preference depending on the future-oriented resources, Stern (2006) assumes a strictly concave discount function.

As a monotone real valued sequence will either diverge to infinity or converge to some real number, the fact that optimal capital sequences are monotone proves to be crucial in analyzing the dynamic properties and the long-run behavior of our model.

Proposition 9. There exists an $\varepsilon>0$ such that if $\sup _{x>0} f^{\prime}(x)<((1-$ $\varepsilon)\left(\beta_{m}\right)$, any optimal path converges to zero.

Proof. Let the optimal path $\mathbf{x}$ converge to $x>0$. Then, by considering the Euler equation as $t \rightarrow \infty$, we obtain

$\frac{u(f(x)-x)}{u^{\prime}(f(x)-x)}=\frac{[1-\beta(x)]\left[1-\beta(x) f^{\prime}(x)\right]}{\beta^{\prime}(x)} \geq \frac{1-\beta_{m}}{\beta_{\text {sup }}^{\prime}}\left[1-\beta_{m} \sup _{x>0} f^{\prime}(x)\right]$,

where $\quad \beta_{\text {sup }}^{\prime} \equiv \sup _{x>0} \beta^{\prime}(x)$. Note that $\quad\left(u / u^{\prime}\right)^{\prime}=\left(\left(\left(u^{\prime}\right)^{2}-\right.\right.$ $\left.\left.u . u^{\prime \prime}\right) /\left(u^{\prime}\right)^{2}\right)>0$, i.e., $u / u^{\prime}$ is increasing. Let $B=\arg \max _{x>0}(f(x)-x)$. In accordance with these, we have:

$\frac{u(f(B)-B)}{u^{\prime}(f(B)-B)} \geq \frac{u(f(x)-x)}{u^{\prime}(f(x)-x)} \geq \frac{1-\beta_{m}}{\beta_{\text {sup }}^{\prime}}\left[1-\beta_{m} \sup _{x>0} f^{\prime}(x)\right]$.

Defining $\varepsilon=\left(\beta_{\text {sup }}^{\prime} /\left(1-\beta_{m}\right)\right)\left((u(f(B)-B)) /\left(u^{\prime}(f(B)-B)\right)\right)$, $\sup _{x>0} f(x) \geq\left(1-\varepsilon / \beta_{m}\right)$ when the optimal path converges to $x>0$. Thus, $\sup _{x>0} f^{\prime}(x)<\left(1-\varepsilon / \beta_{m}\right)$ implies that the optimal path converges to zero.

In a classic optimal growth model, if $f^{\prime}(0) \leq 1 / \beta$, then the optimal path converges to zero. As we consider the endogenous nature of time preference and allow for nonconvexities in the production technology, we have slightly deviated from this condition. However, it must be noted that the interval for $\sup _{x>0} f(x)$ that guarantees the convergence to zero will always be a subset of $(0$, $\left.1 / \beta_{m}\right)$ and this cannot be improved in an essential way.

We will now present the condition under which the convergence to an optimal steady state is guaranteed and analyze the behavior of the optimal paths when we would have unique or multiple optimal steady states.

Proposition 10. Assume $x_{0}>0$. Let $\inf _{x>0} \beta(x)=\beta$. If $f^{\prime}(0)>1 / \beta$, then the optimal path converges to an optimal steady state $x^{s}>0$.

Proof. Since $\beta(\cdot)$ is increasing, $\beta=\beta(0)$. Note that $\left[f^{f} \beta\right](\cdot)$ is a continuous function and $f^{\prime}(0) \beta(0)>1$. Assume that $\mathbf{x}$ is an optimal 
path and it converges to zero. Since $\left[f^{f} \beta\right](\cdot)$ is continuous, there exists $N$ such that $n>N$ implies $f\left(x_{n}\right) \beta\left(x_{n}\right)>1$.

If $\mathbf{x}$ is an optimal path then it satisfies the Euler equation. Hence, for every $t$, in particular, for $t \geq N$, we have:

$$
\begin{aligned}
u^{\prime}\left(f\left(x_{t}\right)-x_{t+1}\right) & =u^{\prime}\left(f\left(x_{t+1}\right)-x_{t+2}\right) \beta\left(x_{t+1}\right) f^{\prime}\left(x_{t+1}\right) \\
& +\beta^{\prime}\left(x_{t+1}\right) V\left(x_{t+1}\right) \\
& >u^{\prime}\left(f\left(x_{t+1}\right)-x_{t+2}\right) \beta\left(x_{t+1}\right) f^{\prime}\left(x_{t+1}\right) \\
& >u^{\prime}\left(f\left(x_{t+1}\right)-x_{t+2}\right),
\end{aligned}
$$

implying that

$f\left(x_{t}\right)-x_{t+1}<f\left(x_{t+1}\right)-x_{t+2}$.

Then, we obtain that:

$0<f\left(x_{N}\right)-x_{N+1}<\lim _{y \rightarrow 0}[f(y)-y]=0$,

leading to a contradiction.

Since the sequence $\mathbf{x}$ is monotonic and bounded, it converges to some $x^{s}>0$. Now $x_{t} \rightarrow x^{s}, x_{t+1} \in \mu\left(x_{t}\right), x_{t+1} \rightarrow x^{s}$. Hence, by the upper semi-continuity of the correspondence $\mu$, we get $x^{s} \in \mu\left(x^{s}\right)$.

With convex technology and exogenously fixed time preference rate in a standard optimal growth model, if $f(0)>1 / \beta$ then there exists a unique (non-zero) steady state to which all optimal paths converge independently of their initial state. In our model, due to the existence of the maximum sustainable level of capital stock, when $f^{\prime}(0)>1 / \beta$, the optimal path converges monotonically towards an optimal steady state. This condition can be recast as $F^{\prime}(0)>r+\delta$ by defining the rate of interest $r$ by $1 /(1+r)=\beta$ and the function $f$ by $f(k)=F(k)+(1-\delta) k$ where $F$ is the production function and $\delta$ is the rate of depreciation of capital. Accordingly, in our model, when the cost of investment is low, the optimal path converges to an optimal steady state.

We will now consider the optimal path dynamics in the long run and show that our model can support unique optimal steady state with global convergence and multiplicity of optimal steady states with local convergence. Indeed, we will show that our model exhibits global convergence even under convex-concave technology and multiplicity of optimal steady states even under convex technology.

Case 1 (Global convergence). Assume $x_{0}>0$. Let inf $x_{x>0} \beta(x)=\beta$ and $f^{\prime}(0)>1 / \beta$. Consider the case where there exists a unique solution $x^{s}$ to (9). By Proposition 10, we know that the optimal paths cannot converge to 0 . Then, any optimal path converges to the unique optimal steady state $x^{s}$, irrespective of their initial state.

When there exists a unique solution to (9), it is clear from above that it will be an optimal steady state. However, when there exist multiple solutions to the steady-state equation (9), the stationary sequences associated with these steady states may or may not be optimal. A natural question is then whether these solutions will constitute a long-run equilibrium in the optimal growth model and if so, will that enable us to show whether an economy with wealth dependent endogenous discounting can exhibit threshold dynamics even under convex technology or not. Devoted to this end, first, we will consider the case where we have exactly two optimal steady states, for the sake of simplicity. We shall prove that this would imply the existence of a critical stock and local convergence. Second, we will show that our model can indeed support two optimal steady states in an example.

Case 2 (Local convergence). Assume $x_{0}>0$. Let $\inf _{x>0} \beta(x)=\beta$ and $f^{\prime}(0)>1 / \beta$. Consider the case where we have exactly two optimal steady states, $x_{l}<x_{h}$, for the sake of simplicity. Suppose that $x_{h}$ is unstable from the right. Given the existence of a maximum sustainable capital stock, as any optimal path from $x_{0}>x_{h}$ has to converge to an optimal steady state, there will be another steady state larger than $x_{h}$, a contradiction. Hence, $x_{h}$ is stable from the right. It is also impossible to have $x_{l}$ unstable from the left, since the optimal paths cannot converge to 0 . These already imply the existence of a critical stock and the emergence of threshold dynamics.

Whenever both of the optimal steady states turn out to be saddle-point stable, it is clear that there will exist a critical stock of capital below which the optimal path will converge to $x_{l}$ and above which the optimal path will converge to $x_{h}$. However, $x_{h}$ can be stable from the right but unstable from the left, and similarly $x_{l}$ can be stable from the left but unstable from the right. These cases can occur only when the optimal policy is tangent to $y=x$ line at $x_{h}$, and $x_{l}$, respectively. It is important to note that even under these cases threshold dynamics emerge. The following proposition provides the formal analysis of the local convergence when we have two optimal steady states.

Proposition 11. Assume $x_{0}>0$. Let inf $_{x>0} \beta(x)=\beta$ and $f^{\prime}(0)>1 / \beta$. Suppose there are exactly two optimal steady states. Let the high and the low optimal steady states be, respectively $x_{h}$ and $x_{l}$. Then there exists $x_{c} \in\left[x_{l}, x_{h}\right]$ such that any optimal path $\mathbf{x}$ starting from $x_{0}$, converges to $x_{l}$ if $x_{0}<x_{c}$, and converges to $x_{h}$ if $x_{0}>x_{c}$.

Proof. Take any initial capital levels $y<z$, and let $\mathbf{y}, \mathbf{z}$ be any two corresponding optimal paths. Since the optimal correspondence is increasing, we iteratively obtain $y_{t}<z_{t}$ for all $t$. Hence $\lim _{t \rightarrow \infty} y_{t} \leq \lim _{t \rightarrow \infty} z_{t}$. Thus, if $\mathbf{z}$ converges to $x_{l}$, so does $\mathbf{y}$. If $\mathbf{y}$ converges to $x_{h}$, so does $\mathbf{z}$. Let $X_{1}=\{x \mid$ there exists an optimal path from $x$ that converges to $\left.x_{l}\right\}, x_{1}^{*}=\sup X_{1}$, and $X_{2}=\{x \mid$ there exists an optimal path from $x$ that converges to $\left.x_{h}\right\}, x_{2}^{*}=\inf X_{2}$. From the above argument, $x_{1}^{*} \leq x_{2}^{*}$. Suppose otherwise, then there exists small $\epsilon_{1}, \epsilon_{2} \geq 0$ such that $x_{1}^{*}-\epsilon_{1} \in X_{1}, x_{2}^{*}+\epsilon_{2} \in X_{2}$, and $x_{1}^{*}-\epsilon_{1}>$ $x_{2}^{*}+\epsilon_{2}$. However, this contradicts with the above, by setting $x_{2}^{*}+$ $\epsilon_{2}=y<z=x_{1}^{*}-\epsilon_{1}$. Now we want to show that $x_{1}^{*}=x_{2}^{*}$. Suppose not, then there exists $x_{3}^{*}$ such that $x_{1}^{*}<x_{3}^{*}<x_{2}^{*}$. But then an optimal path from $x_{3}^{*}$ can neither converge to $x_{l}$ nor to $x_{h}$ by the definitions of $x_{1}^{*}$ and $x_{2}^{*}$. Therefore, $x_{1}^{*}=x_{2}^{*}$. Define $x_{c}=x_{1}^{*}=x_{2}^{*}$. If $x_{0}<x_{c}=x_{1}^{*}$, take some small $\epsilon_{1}^{\prime} \geq 0$ with $x_{0}<x_{1}^{*}-\epsilon_{1}^{\prime} \in X_{1}$. By the above argument, any optimal path from $x_{0}$ converges to $x_{1}$. Similarly, if $x_{0}>x_{c}=x_{2}^{*}$, take some small $\epsilon_{2}^{\prime} \geq 0$ with $x_{0}>x_{2}^{*}+\epsilon_{2}^{\prime} \in X_{2}$, which would show that any optimal path from $x_{0}$ converges to $x_{h}$. Notice that $x_{l} \in X_{1}$ and $x_{h} \in X_{2}$. Therefore, $x_{l} \leq \sup X_{1}=x_{1}^{*}=x_{c}=$ $x_{2}^{*}=\inf X_{2} \leq x_{h}$, i.e., $x_{c} \in\left[x_{l}, x_{h}\right]$.

Remark 1. As it has become clear by now, if $\mu$ is not tangent to $y=x$ line at either $x_{l}$ or $x_{h}$, both are locally stable and $x_{c} \in\left(x_{l}, x_{h}\right)$. Note that if $\mu$ is not tangent to $y=x$ line at either $x_{l}$ or $x_{h}$, it must be the case that $\mu$ jumps over $y=x$ line at $x_{c}$. In this case, $x_{c}$ is not an unstable optimal steady state but a genuine critical point leading to the threshold dynamics.

If $\mu$ is tangent to $y=x$ line at $x_{l}$, it can only be from above the $y=x$ line because $x_{l}$ is stable from the left. In this case we get $x_{c}=x_{l}$. Similarly, if $\mu$ is tangent to $y=x$ line at $x_{h}$, it can only be from below the $y=x$ line and we get $x_{C}=x_{h}$.

In order to provide a better exposition of our analysis, following Stern (2006), we will specify functional forms in an example and show that our model can indeed support two optimal steady states with local convergence. 


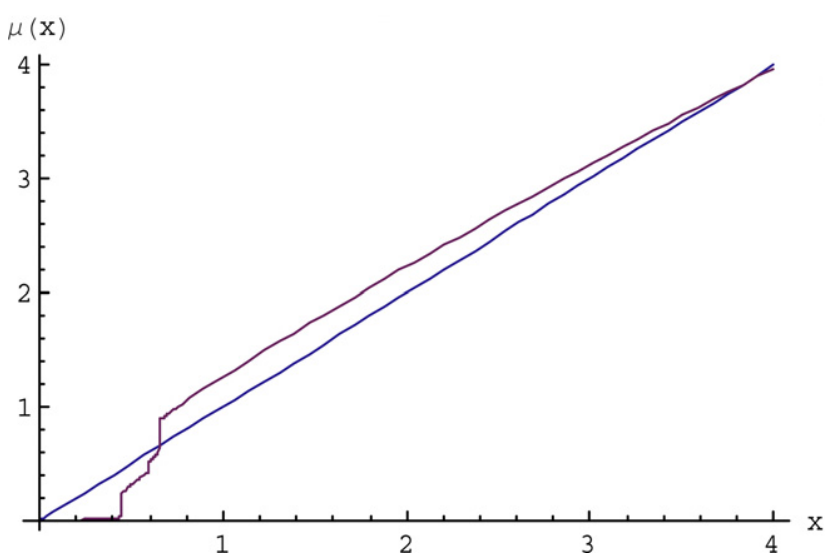

Fig. 1. Optimal policy after 300 iterations on the initial zero value function.

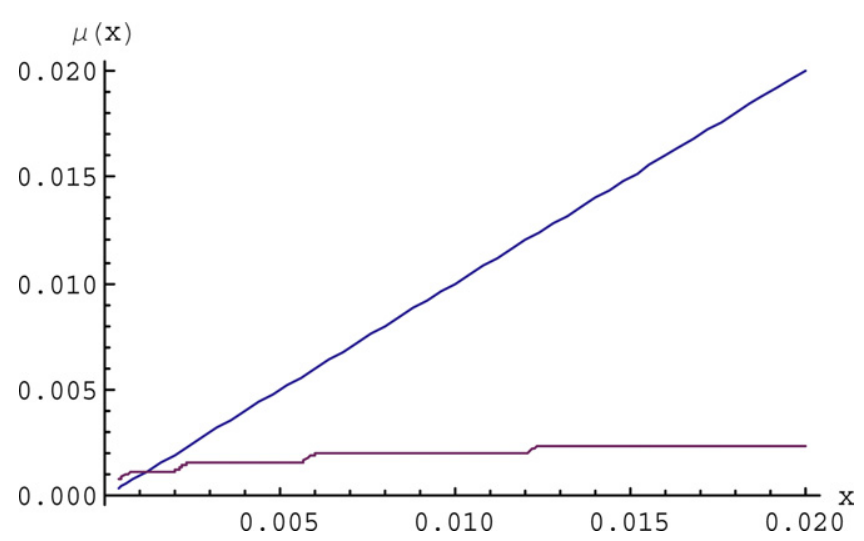

Fig. 2. Low steady state $=0.0012$ is optimal.

Example 1. Suppose that

$u(c)=\frac{c^{1-\sigma}}{1-\sigma}$,
$f(x)=A x^{\alpha}+(1-\delta) x$,
$\beta(x)=\eta-\gamma e^{-(x+\rho)^{\varepsilon}}$,

where $0<\{A, \rho\}, 0<\{\alpha, \sigma, \varepsilon\}<1,0<\gamma<e^{\rho^{\varepsilon}}$, and $\gamma e^{-\rho^{\varepsilon}}<\eta<$ 1 . Check that $f, u$, and $\beta$ satisfy the assumption sets. We employ the following set of fairly standard coefficients:

$A=0.5, \quad \alpha=0.3, \quad \delta=0.15, \quad \sigma=0.5, \quad \eta=0.97, \quad \gamma=2.5$,

$$
\rho=1, \quad \varepsilon=0.9 \text {. }
$$

It turns out that the maximum sustainable capital stock is $A\left(x_{0}\right)=$ $\max \left\{x_{0}, \bar{x}\right\}$, where $\bar{x}$ is 5.58431, and there exist three solutions to (9). The precise values are, $x_{l}=0.0012, x_{m}=0.5865$, and $x_{h}=3.8105$. In order to determine which of these are actually the optimal steady states, we analyze the optimal policy by making use of Bellman's operator. Fig. 1 shows the optimal policy for iterations of the Bellman operator on the zero function and indicates that $x_{l}$ and $x_{h}$ are stable optimal steady states. Figs. 2-4 present the detailed pictures of the optimal policy in the neighborhood of the $x_{l}, x_{m}$ and $x_{h}$, respectively.

At first sight, one might think that the middle steady state $x_{m}$ is an unstable optimal steady state as it is surrounded by two stable optimal steady states. In contrast with Stern (2006), even though $x_{m}$ is a solution of the stationary Euler equation (9), Fig. 1 strongly indicates that it is not an optimal steady state. Indeed, if it were, $\mu$

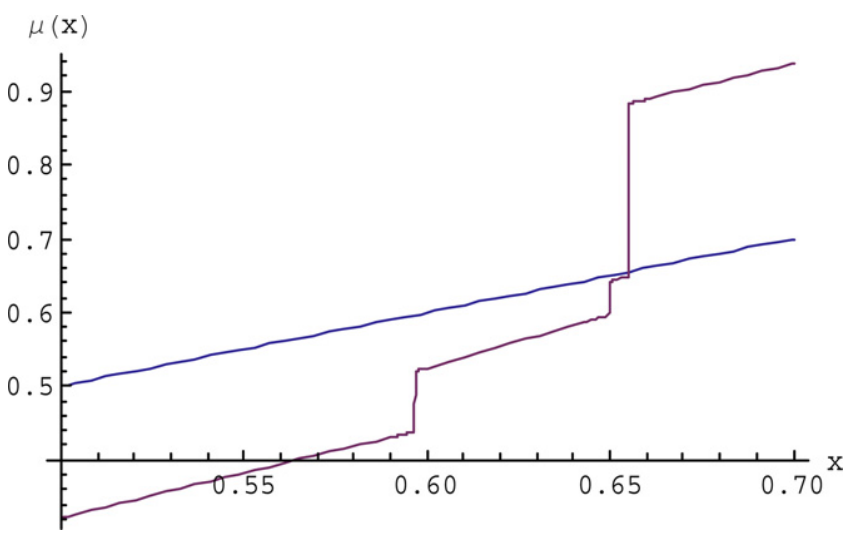

Fig. 3. Middle steady state $\left(x_{m}=0.586505\right)$ is not optimal!

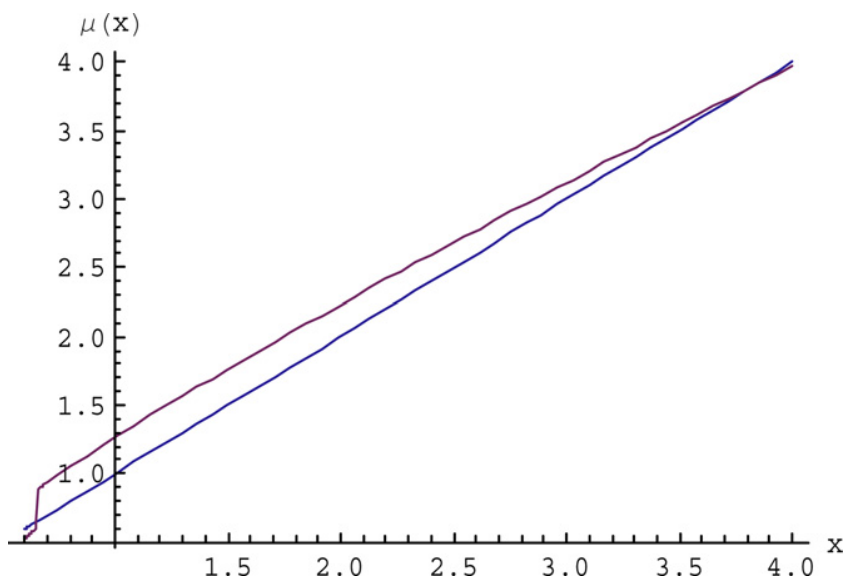

Fig. 4. High steady state $\left(x_{h}=3.81057\right)$ is optimal.

would have to cross $y=x$ line at $x_{m}$. Moreover, we see that there is genuine critical point at $x_{c}=0.6548$. It is clearly not an optimal or nonoptimal steady state because then it would have to satisfy (9).

As $x_{C}$ is not an optimal steady state, $\mu$ cannot cross $y=x$ line at $x_{c}$, i.e., $x_{c} \notin \mu\left(x_{c}\right)$. On the other hand, Fig. 1 shows that the graph of $\mu$ jumps over $y=x$ line at $x_{c}$. As $\mu$ is upper semi continuous, it must be the case that there exist $x_{1}^{\prime} \in\left(0, x_{c}\right) \cap \mu\left(x_{c}\right)$ and $x_{1}^{\prime \prime} \in\left(x_{c}, \infty\right) \cap \mu\left(x_{c}\right)$. Therefore, $x_{c}$ is a critical point which is not an unstable steady state.

For any initial capital stock level lower than $x_{c}$, the system will face a development trap, enforcing convergence to a very low capital level $x_{l}$, even under a strictly concave production function. On the other hand, for any initial capital level higher than $x_{c}$, the optimal path will converge to $x_{h}$. However, if an economy starts at $x_{c}$, an indeterminacy will emerge: the system can optimally follow $x_{1}^{\prime \prime}$, and at the same time it can optimally fall into a development trap following $x_{1}^{\prime}$.

The existence of critical value is actually recognized since the papers by Clark (1971); Majumdar and Mitra (1982); Dechert and Nishimura (1983) in discrete time and Skiba (1978) and Askenazy and Le Van (1999) in continuous time horizon. These studies are mostly devoted to the analysis of the technology component leaving the time preference essentially unaltered with an exogenously fixed geometric discounting. They assume a specific convex-concave technology under which the low steady state turns out to be unstable and high steady state turns out to be stable so that an optimal path converges either to zero or to the high steady state. However, we show that even under strictly concave production function, the economy can exhibit a "trap" so that a critical value of the initial stock will exist, in the vicinity of which, 
small differences will lead to permanent differences in the optimal path.

In this respect, our analysis is in line with the early contributions that emphasize the importance of the subjective utility function and release the optimal growth theory from the almost taken for granted Ramsey's additive utility function (e.g., Beals and Koopmans, 1969; Iwai, 1972). Based upon the stationary ordinal utility function that implies a broad class of preference structures that are recursive and contain the time-additive preference as a special case, they show the existence of a critical stock so that the initial conditions can affect the long-run optimal path. Adopting a strictly concave discount function depending on the future-oriented resources, Stern (2006) analyzes a series of numerical examples that also exhibit multiplicity of steady states with mere local convergence. Besides these, Kurz (1968) introduces capital as an argument in the Ramsey type utility function and shows also that there may exist multiple turnpikes.

In all of these studies mentioned above, the critical stock of capital below which the optimal program leads only to forever diminishing capital and gradually converging to development trap is an unstable optimal steady state. If it so happens that the initial level of capital stock equals the critical stock, the optimal path will remain on that generalized turnpike forever. However, in our model the critical stock is definitely not an unstable steady state so that if an economy starts at this stock, an indeterminacy emerges. Compared to the optimal growth models with exogenous time preference, this introduces a fundamental difference in the optimal path dynamics.

\section{Competitive equilibrium with externality}

From now on, we will assume that the production function $f$ is strictly concave. We first define the concepts of equilibrium with externality and competitive equilibrium. Suppose we are given a sequence of capital $\tilde{\boldsymbol{x}}=\left(x_{0}, \tilde{x}_{1}, \ldots, \tilde{x}_{t}, \ldots\right) \in\left[0, A\left(x_{0}\right)\right]^{\infty}$ and the associated sequence of discount factors $\tilde{\beta}=\left(\tilde{\beta}_{1}, \ldots, \tilde{\beta}_{t}, \ldots\right)$ where $\tilde{\beta}_{t}=\beta\left(\tilde{x}_{t}\right), \forall t \geq 1$. Given this fixed sequence $\tilde{\boldsymbol{\beta}} \in\left[\underline{\beta}, \beta_{m}\right]$, consider the following problem:

$\max _{\mathbf{x}} \sum_{t=0}^{\infty}\left(\prod_{s=1}^{t} \tilde{\beta}_{s}\right) u\left(f\left(x_{t}\right)-x_{t+1}\right)$

subject to

$\forall t, 0 \leq x_{t+1} \leq f\left(x_{t}\right)$,

$x_{0}>0$,given.

Let the solution to the above problem be $\mathbf{x}=\left(x_{0}, x_{1}, \ldots, x_{t}\right.$, $\ldots)$. It depends on $\tilde{\beta}$, hence $\tilde{\mathbf{x}}$. We write $\mathbf{x}=\psi(\tilde{\boldsymbol{\beta}})=\boldsymbol{\psi}(\beta(\tilde{\mathbf{x}}))$, and hence $\mathbf{x}=\boldsymbol{\Phi}(\tilde{\mathbf{x}})$. An equilibrium with externality associated with $x_{0}$ is a sequence of capital stock $\mathbf{x}^{*}=\left(x_{0}, x_{1}^{*}, \ldots, x_{t}^{*}, \ldots\right)$ such that $\mathbf{x}^{*}=\boldsymbol{\Phi}\left(\mathbf{x}^{*}\right)$. A list of sequences $\left(\mathbf{x}^{*}, \mathbf{c}^{*}, \mathbf{p}^{*}, q^{*}\right)$ is a competitive equilibrium with externality of this economy if the following are satisfied:

(a) $\mathbf{c}^{*} \in \ell_{+}^{\infty}, \mathbf{x}^{*} \in \ell_{+}^{\infty}, \mathbf{p}^{*} \in\left(\ell_{+}^{1} \backslash\{\mathbf{0}\}\right), q^{*} \in \mathbb{R}_{++}$,

(b) $\mathbf{c}^{*}$ solves the consumer's problem:

$$
\max _{\mathbf{c}} \sum_{t=0}^{\infty}\left(\prod_{s=1}^{t} \beta\left(x_{s}^{*}\right)\right) u\left(c_{t}\right)
$$

subject to

$$
\sum_{t=0}^{\infty}{ }_{t}^{*} c_{t} \leq q^{*} x_{0}+\pi^{*}
$$

where $\pi^{*}$ is the maximum profit of the firm, (c) $\mathbf{x}^{*}$ solves the firm's problem:

$$
\pi^{*}=\max _{\mathbf{x}} \sum_{t=0}^{\infty} p_{t}^{*}\left(f\left(x_{t}\right)-x_{t+1}\right)-q^{*} x_{0}
$$

subject to

$\forall t \geq 0,0 \leq x_{t+1} \leq f\left(x_{t}\right)$,

$x_{0}>0$,given,

(d) the markets clear at every period:

$$
\forall t \geq 0, c_{t}^{*}+x_{t+1}^{*}=f\left(x_{t}^{*}\right), x_{0}^{*}=x_{0} .
$$

The purpose is to prove the existence of a competitive equilibrium of this intertemporal economy. In order to do so, we will first prove that there exists a fixed point of $\boldsymbol{\Phi}$, namely an equilibrium. Later, under an additional assumption, we will show that this equilibrium is indeed a competitive equilibrium of the intertemporal economy with endogenous time preference.

The value function associated with the problem $(\mathcal{P E})$ takes the following form:

$V\left(x_{0}, \beta_{1}, \beta_{2}, \beta_{3}, \ldots\right)=\max _{\mathbf{x}} \sum_{t=0}^{\infty}\left(\prod_{s=1}^{t} \beta_{s}\right) u\left(f\left(x_{t}\right)-x_{t+1}\right)$

subject to

$0 \leq x_{t+1} \leq f\left(x_{t}\right)$,

$x_{0}>0$,given.

$V$ satisfies the Bellman equation:

$V\left(x, \beta_{t+1}, \beta_{t+2}, ..\right)=\max _{y \in[0, f(x)]}\left[u(f(x)-y)+\beta_{t+1} V\left(y, \beta_{t+2}, \beta_{t+3}, ..\right)\right]$.

Proposition 12. The function $V$ is continuous with the topology in $\mathbb{R}$ for $x_{0}$ and the product topology for $\boldsymbol{\beta} \in\left[\underline{\beta}, \beta_{m}\right]^{\infty}$. Moreover, it is strictly concave in $x_{0}$

Proof. Let

$U\left(x_{0}, \boldsymbol{\beta}, \mathbf{x}\right)=\sum_{t=0}^{\infty}\left(\prod_{s=1}^{t} \beta_{s}\right) u\left(f\left(x_{t}\right)-x_{t+1}\right)$

where $\mathbf{x} \in \Pi\left(x_{0}\right)$. One can show that $U$ is continuous in $\left(x_{0}, \boldsymbol{\beta}, \mathbf{x}\right)$ for the topology in $\mathbb{R}$ for $x_{0}$ and the product topology for $(\boldsymbol{\beta}, \mathbf{x})$. It is well known that $\Pi(\cdot)$ is a continuous correspondence from $\mathbb{R}_{+}$into the space of sequences endowed with the product topology (see, for instance, Le Van and Dana, 2003). Since

$V\left(x_{0}, \boldsymbol{\beta}\right)=\max \left\{U\left(x_{0}, \boldsymbol{\beta}, \mathbf{x}\right) \mid \mathbf{x} \in \Pi\left(x_{0}\right)\right\}$,

from the maximum theorem, $V$ is continuous. The strict concavity of $V$ with respect to $x_{0}$ follows from the strict concavity of $f$ and $u$.

Lemma 2. The map $\boldsymbol{\beta}$ is continuous with respect to the product topology.

Proof. It is standard.

Proposition 13. $\Phi$ is continuous with respect to the product topology.

Proof. From the maximum theorem, the solution $\mathbf{x}=\psi(\boldsymbol{\beta})$ is upper semi-continuous with respect to $\beta$. As the solution to $(\mathcal{P E})$ is unique, it is a continuous function with respect to $\boldsymbol{\beta}$. Since $\tilde{\boldsymbol{\beta}}=\boldsymbol{\beta}(\tilde{\mathbf{x}})$, the map $\boldsymbol{\Phi}$ is a continuous function with respect to $\tilde{\mathbf{x}}$ by Lemma 2 . 
To attain an equilibrium, the initial sequence of discounting has to be consistent with the level that is assumed when the agent makes her single decisions. This suggests that the fixed points of $\boldsymbol{\Phi}$ are candidates for competitive equilibria.

\section{Proposition 14. $\Phi$ has a fixed point.}

Proof. $\boldsymbol{\Phi}$ is a continuous mapping from $\left[0, A\left(x_{0}\right)\right]^{\infty}$ into $\left[0, A\left(x_{0}\right)\right]^{\infty}$. Since the domain is compact for the product topology and also a convex set, Schauder theorem concludes that there exists $\mathbf{x}^{*} \in[0$, $\left.A\left(x_{0}\right)\right]^{\infty}$ which satisfies $\mathbf{x}^{*}=\Phi\left(\mathbf{x}^{*}\right)$.

Proposition 15. If $\left(x_{0}, x_{1}^{*}, x_{2}^{*} \ldots\right)$ is an equilibrium with externality associated with $x_{0}$, then $\left(x_{1}^{*}, x_{2}^{*}, x_{3}^{*} \ldots\right)$ is an equilibrium with externality associated with $x_{1}^{*}$.

Proof. Let $V\left(x, \beta_{t+1}, \beta_{t+2}, ..\right)$ be the value function at period $t+1$. For any $t$ we have

$$
\begin{aligned}
V\left(x_{t}^{*}, \beta_{t+1}, \beta_{t+2}, . .\right) & =\max _{y \in[0, f(x)]} \\
& {\left[u(f(x)-y)+\beta_{t+1} V\left(y, \beta_{t+2}, \beta_{t+3}, . .\right)\right] } \\
& =u\left(f\left(x_{t}^{*}\right)-x_{t+1}^{*}\right)+\beta_{t+1} V\left(x_{t+1}^{*}, \beta_{t+2}, \beta_{t+3}, . .\right)
\end{aligned}
$$

Particularly for $t \geq 2$, we see that $\left(x_{1}^{*}, x_{2}^{*}, \ldots\right)$ is an equilibrium with externality associated with $x_{1}^{*}$.

Proposition 16. Any equilibrium with externality $\mathbf{x}^{*}$ associated with some initial capital stock satisfies the Euler equation:

$u^{\prime}\left(f\left(x_{t}^{*}\right)-x_{t+1}^{*}\right)=u^{\prime}\left(f\left(x_{t+1}^{*}\right)-x_{t+2}^{*}\right) \beta\left(x_{t+1}^{*}\right) f^{\prime}\left(x_{t+1}^{*}\right)$.

Proof. It is easy.

For the rest, we assume that the function $\left[\beta f^{\prime}\right](\cdot)$ is decreasing and $\left[\beta f^{\prime}\right](0)>1$. Note that $\lim _{x \rightarrow \infty}\left[\beta f^{\prime}\right](x)<1$.

Proposition 17. The equilibrium with externality associated with $x_{0}$ is unique.

Proof. Take two equilibria with externality $\left(x_{0}, x_{1}^{\prime}, x_{2}^{\prime}, \ldots\right)$ and $\left(x_{0}, x_{1}^{\prime \prime}, x_{2}^{\prime \prime}, \ldots\right)$ associated with $x_{0}$. Suppose that $x_{1}^{\prime}>x_{1}^{\prime \prime}$. Let the associated consumption paths be $\left(c_{0}^{\prime}, c_{1}^{\prime}, \ldots\right)$ and $\left(c_{0}^{\prime \prime}, c_{1}^{\prime \prime}, \ldots\right)$.

First, we will prove by induction that $x_{t}^{\prime}>x_{t}^{\prime \prime}$, for all $t \geq 1$ and $c_{t}^{\prime}<c_{t}^{\prime \prime}$ for all $t$. Trivially, $c_{0}^{\prime}=f\left(x_{0}\right)-x_{1}^{\prime}<f\left(x_{0}\right)-x_{1}^{\prime \prime}=c_{0}^{\prime \prime}$. Then by (10), $u^{\prime}\left(c_{1}^{\prime}\right)\left[\beta f^{\prime}\right]\left(x_{1}^{\prime}\right)=u^{\prime}\left(c_{0}^{\prime}\right)>u^{\prime}\left(c_{0}^{\prime \prime}\right)=u^{\prime}\left(c_{1}^{\prime \prime}\right)\left[\beta f^{\prime}\right]\left(x_{1}^{\prime \prime}\right)$ which implies $u^{\prime}\left(c_{1}^{\prime}\right)>u^{\prime}\left(c_{1}^{\prime \prime}\right)$ as $\left[\beta f^{\prime}\right]$ is a decreasing function. Hence, $c_{1}^{\prime}<$ $c_{1}^{\prime \prime}$.

Now suppose $x_{t}^{\prime}>x_{t}^{\prime \prime}$ and $c_{t}^{\prime}<c_{t}^{\prime \prime}$ for some $t$. $f\left(x_{t}^{\prime}\right)-$ $x_{t+1}^{\prime}=c_{t}^{\prime}<c_{t}^{\prime \prime}=f\left(x_{t}^{\prime \prime}\right)-x_{t+1}^{\prime \prime}$, so $x_{t+1}^{\prime}>x_{t+1}^{\prime \prime}$. Again, by (10), $u^{\prime}\left(c_{t+1}^{\prime}\right)\left[\beta f^{\prime}\right]\left(x_{t+1}^{\prime}\right)=u^{\prime}\left(c_{t}^{\prime}\right)>u^{\prime}\left(c_{t}^{\prime \prime}\right)=u^{\prime}\left(c_{t+1}^{\prime \prime}\right)\left[\beta f^{\prime}\right]\left(x_{t+1}^{\prime \prime}\right) \quad$ which implies $u^{\prime}\left(c_{t+1}^{\prime}\right)>u^{\prime}\left(c_{t+1}^{\prime \prime}\right)$ as $\left[\beta f^{\prime}\right]$ is a decreasing function. Hence, $x_{t+1}^{\prime}>x_{t+1}^{\prime \prime}$ and $c_{t+1}^{\prime}<c_{t+1}^{\prime \prime}$.

Now consider the maximization problem given the discounting sequence $\beta\left(\mathbf{x}^{\prime}\right)$. The path $\mathbf{x}^{\prime \prime}$ is feasible from $x_{0}$, and yields strictly higher utility than $\mathbf{x}^{\prime}$ given the discounting sequence $\beta\left(\mathbf{x}^{\prime}\right)$ because $c_{t}^{\prime}<c_{t}^{\prime \prime}$ for all $t$. However, this is a contradiction with $\mathbf{x}^{\prime}$ being a fixed point which would imply that $\mathbf{x}^{\prime}$ itself is the unique maximizer given the discounting sequence $\beta\left(\mathbf{x}^{\prime}\right)$. Therefore $x_{1}^{\prime}=x_{1}^{\prime \prime}$. Let $x_{1}=$ $x_{1}^{\prime}=x_{1}^{\prime \prime}$.

Notice that Proposition 15 implies that $\left(x_{1}, x_{2}^{\prime}, x_{3}^{\prime}, \ldots\right)$ and $\left(x_{1}, x_{2}^{\prime \prime}, x_{3}^{\prime \prime}, \ldots\right)$ are two equilibria with externality associated with $x_{1}$. Then applying the same arguments, we get $x_{2}^{\prime}=x_{2}^{\prime \prime}$. Finally, by inductively applying Proposition 15 and the above arguments, we get $x_{t}^{\prime}=x_{t}^{\prime \prime}$ for all $t$.

Corollary 1. If $\left[\beta f^{\prime}\right]\left(x_{0}\right)=1$ for some $x_{0}$, then the unique fixed point associated with $x_{0}$ is the stationary sequence $\left(x_{0}, x_{0}, x_{0}, \ldots\right)$.

Proof. Let $\beta=\beta\left(x_{0}\right)$ and consider the basic neoclassical model with fixed discount factor $\beta$. As the initial capital is already $x_{0}$ which is steady state capital level in the neoclassical growth model, $\left(x_{0}, x_{0}\right.$, $\left.x_{0}, \ldots\right)$ will solve the social planner problem. This means that $\left(x_{0}\right.$, $\left.x_{0}, x_{0}, \ldots\right)$ is the unique maximizer given the discount sequence $(\beta, \beta, \ldots)$. Thus, it is a fixed point associated with $x_{0}$, and by the above proposition it is the unique fixed point associated with $x_{0}$.

Proposition 18. Any equilibrium with externality monotonically converges to a point $x^{s}$ satisfying $\left[\beta f^{f}\right]\left(x^{s}\right)=1$. Moreover, if there are multiple such points, it monotonically converges to the one that is closest to the initial level of capital.

Proof. One can show that the set of solutions to $[\beta f](x)=1$ constitues a compact interval, say $X=\left[x_{\min }^{s}, x_{\max }^{s}\right]$. Note that it is well possible to have $x_{\min }^{s}=x_{\max }^{s}$ or $x_{\min }^{s}<x_{\max }^{s}$. Let the initial capital level be $x_{0}$ and the associated equilibrium with externality be $\mathbf{x}^{*}$.

By Corollary 1 , if $x_{0} \in X$ we have $\mathbf{x}^{*}=\left(x_{0}, x_{0, \ldots}\right)$ which converges to the closest solution $x_{0}$ to $\left[\beta f^{\prime}\right](x)=1$.

Consider $x_{0}<x_{\min }^{s}$. Suppose $\mathbf{x}^{*}$ does not - monotonically converge - to $x_{\min }^{s}$. Then $\mathbf{x}^{*}$ as a sequence either passes from the region $\left(0, x_{\min }^{s}\right]$ to the region $\left(x_{\min }^{s}, \infty\right)$, or strictly decreases in the region $\left[0, x_{\min }^{s}\right]$, at least once at some point in time. Formally, there exists some $t$ either with $x_{t}^{*} \leq x_{\min }^{s}<x_{t+1}^{*}$ or with $x_{t+1}^{*}<x_{t}^{*} \leq x_{\min }^{s}$.

Suppose that for some $t, x_{t} \leq x_{\min }^{s}<x_{t+1}$. As $1=\left[\beta f^{\prime}\right]\left(x_{\min }^{s}\right) \geq$ $\left[\beta f^{\prime}\right]\left(x_{t+1}^{*}\right),(10)$ implies $u^{\prime}\left(c_{t}^{*}\right) \leq u^{\prime}\left(c_{t+1}^{*}\right)$. Then by concavity of $u$ we get $f\left(x_{t}^{*}\right)-x_{t+1}^{*} \geq f\left(x_{t+1}^{*}\right)-x_{t+2}^{*}$, which yields $x_{t+1}^{*}<x_{t+2}^{*}$ as $x_{t}^{*}<$ $x_{t+1}^{*}$. Hence, $x_{t+1}^{*}<x_{t+2}^{*}$ and $x_{\min }^{s}<x_{t+2}^{*}$. Inductively we obtain $x_{\min }^{s}<x_{t+1}^{*}<x_{t+2}^{*}<\ldots$. Due to the maximum sustainable capital stock, $\mathbf{x}^{*}$ cannot diverge, so it converges monotonically to a level $x$ that is strictly higher than $x_{t+1}^{*}$. Taking the limit of (10) for the fixed point $\mathbf{x}^{*}$ as $t$ goes to infinity, we see that the limit $x$ of $\mathbf{x}^{*}$ has to satisfy $\left[\beta f^{f}\right](x)=1$. Thus $x_{\min }^{s}<x_{t+1}^{*}<x \leq x_{\max }^{s}$ and hence $x_{t+1}^{*} \in X$. Then Corollary 1 implies that the equilibrium with externality associated with $x_{t+1}^{*}$ is $\left(x_{t+1}^{*}, x_{t+1}^{*}, \ldots\right)$, however, Proposition 15 implies that it is $\left(x_{t+1}^{*}, x_{t+2}^{*}, \ldots\right)$. Recall that the fixed point from $x_{t+1}^{*}$ is unique, so we get a contradiction as $x_{t+1}^{*}<x_{t+2}^{*}$.

Now suppose that for some $t, x_{t+1}^{*}<x_{t}^{*} \leq x_{\min }^{s}$. As $1=$ $\left[\beta f^{\prime}\right]\left(x_{\text {min }}^{s}\right) \leq\left[\beta f^{\prime}\right]\left(x_{t+1}^{*}\right),(10)$ implies $u^{\prime}\left(c_{t}^{*}\right) \geq u^{\prime}\left(c_{t+1}^{*}\right)$. Thus we get $f\left(x_{t}^{*}\right)-x_{t+1}^{*} \leq f\left(x_{t+1}^{*}\right)-x_{t+2}^{*}$, which yields $x_{t+1}^{*}>x_{t+2}^{*}$ as $x_{t}^{*}>x_{t+1}^{*}$. Hence, $x_{t+2}^{*}<x_{t+1}^{*}<x_{\min }^{s}$. Inductively we obtain $\ldots<x_{t+1}^{*}<x_{t}^{*} \leq$ $x_{\min }^{s}$. Taking the limit of (10), we see that the only possibility is $\mathbf{x}^{*}$ converges to zero. If $\mathbf{x}^{*}$ converges to zero, there exists some large $T$ such that $\left[\beta f^{\prime}\right]\left(x_{t}^{*}\right)>1$ for all $t>T$, because $\left[\beta f^{\prime}\right](0)>1$. Then $u^{\prime}\left(c_{t}^{*}\right)>$ $u^{\prime}\left(c_{t+1}^{*}\right)$ for $t>T$ by (10). Hence, $f\left(x_{t}^{*}\right)>f\left(x_{t}^{*}\right)-x_{t+1}^{*}=c_{t}^{*}>c_{T}^{*}>0$ for all $t>T$. However, $f\left(x_{t}^{*}\right)$ converges to zero along with $\mathbf{x}^{*}$ by the continuity of $f$. Contradiction.

Therefore we have shown that the fixed point associated with $x_{0}<x_{\min }^{s}$ monotonically converges to the closest solution $x_{\min }^{s}$ of the equation $[\beta f](x)=1$.

The case of $x_{0}>x_{\max }^{s}$ is analogous to $x_{0}<x_{\min }^{s}$.

Just to recall, the fixed point $\mathbf{x}^{*}$ of $\boldsymbol{\Phi}$, namely the equilibrium with externality associated with $x_{0}$, solves the problem

$\max _{\mathbf{x}} \sum_{t=0}^{\infty}\left(\prod_{s=1}^{t} \beta\left(x_{s}^{*}\right)\right) u\left(f\left(x_{t}\right)-x_{t+1}\right)$

subject to

$0 \leq x_{t+1} \leq f\left(x_{t}\right)$,

$x_{0}>0$,given.

We will now prove that such a fixed point is indeed a competitive equilibrium.

Theorem 1. Assume that $f^{\prime}(0) \underline{\beta}>1$. Define $q^{*}=1, p_{t}^{*}=$ $\left(\prod_{s=1}^{t} \beta\left(x_{s}^{*}\right)\right) u^{\prime}\left(f\left(x_{t}^{*}\right)-x_{t+1}^{*}\right), \quad c_{t}^{*}=f\left(x_{t}^{*}\right)-x_{t+1}^{*}, \forall t$. Then, $\left(\mathbf{c}^{*}, \mathbf{x}^{*}\right.$, $\left.\mathbf{p}^{*}, q^{*}\right)$ is a competitive equilibrium with externality. 
Proof. First, we prove that $\mathbf{p}^{*}$ is in $\ell_{+}^{1} \backslash\{\mathbf{0}\}$. Clearly $p_{t}^{*}>$ 0. We need to prove that $\sum_{t=0}^{\infty} p_{t}^{*}=\sum_{t=0}^{\infty}\left(\prod_{s=1}^{t} \beta\left(x_{s}^{*}\right)\right) u^{\prime}\left(f\left(x_{t}^{*}\right)-x_{t+1}^{*}\right)$ is bounded. We have shown that $\mathbf{x}^{*}$ converges to some $x^{s} \in X$. $\left[\beta f^{\prime}\right](0)>1$ suggests $x_{\text {min }}^{s}>0$, hence the sequence $u^{\prime}\left(f\left(x_{t}^{*}\right)-x_{t+1}^{*}\right)$ is bounded. Thus, the sum $\sum_{t=0}^{\infty} p_{t}^{*}$ exists in $\mathbb{R}_{+}$.

Since $\mathbf{x}^{*} \in\left[0, A\left(x_{0}\right)\right]^{\infty}$ we get $\mathbf{x}^{*} \in \ell_{+}^{\infty}$. By the constraints of the problem $(\mathcal{P E}), f\left(A\left(x_{0}\right)\right) \geq f\left(x_{t}^{*}\right) \geq x_{t+1}^{*} \geq 0$. Hence, $f\left(A\left(x_{0}\right)\right) \geq c_{t}^{*}=$ $f\left(x_{t}^{*}\right)-x_{t+1}^{*} \geq 0$, i.e., $\mathbf{c}^{*} \in \ell_{+}^{\infty}$.

The proofs that $\mathbf{x}^{*}$ is a solution to the problem $(\mathcal{P P})$ and $\mathbf{c}^{*}$ solves the consumer's problem $(\mathcal{C P})$ are standard (see, e.g., Le Van and Dana, 2003).

Finally, one can easily note that the market clearing condition, $c_{t}^{*}+x_{t+1}^{*}=f\left(x_{t}^{*}\right)$ is satisfied.

By Proposition 18, the optimal paths converge to a point $x^{*}$ with $f^{\prime}\left(x^{*}\right) \beta\left(x^{*}\right)=1$.

Clearly, such a point is unique if $\left[\beta f^{\prime}\right](\cdot)$ is strictly decreasing.

For simplicity, let us assume a Cobb-Douglas production technology, $f(x)=A x^{\alpha}$, where $A>0$ denotes the total factor productivity. There may exist unique or continuum of steady states in this model with endogenous time preference depending on the characteristics of the discount function.

Example 2. Let $\beta(x)=\theta-\gamma e^{-(x+\rho)^{\varepsilon}}$, where $0<\rho, 0<\varepsilon<1,0<\gamma<$ $(1-\alpha) e^{\rho^{\varepsilon}}$, and $\left(\gamma e^{-\rho^{\varepsilon}} / 1-\alpha\right)<\theta<1$. Note that $\beta$ satisfies all of our assumptions. We will now show that $\beta(x) f^{\prime}(x)$ is strictly decreasing. We have

$$
\begin{aligned}
& \left(\beta(x) f^{\prime}(x)\right)^{\prime}=\left[\left(\theta-\gamma e^{-(x+\rho)^{\varepsilon}}\right) A \alpha x^{\alpha-1}\right]^{\prime} \\
& =A \alpha x^{\alpha-2}\left[\gamma e^{-(x+\rho)^{\varepsilon}}\left(1-\alpha+\varepsilon x(x+\rho)^{\varepsilon-1}\right)-(1-\alpha) \theta\right] .
\end{aligned}
$$

Then, for $x>0,\left(\beta(x) f^{\prime}(x)\right)^{\prime}<0$ if and only if

$h(x):=\gamma \exp \left\{-(x+\rho)^{\varepsilon}\right\}\left[1-\alpha+\varepsilon x(x+\rho)^{\varepsilon-1}\right]-(1-\alpha) \theta<0$.

It is clear that $h$ is differentiable, hence obtains its maximum at the boundaries or critical points. At the boundaries,

$$
\begin{aligned}
h(0)= & \gamma \exp \left(-\rho^{\varepsilon}\right)[1-\alpha]-(1-\alpha) \theta \\
& =(1-\alpha)\left[\gamma \exp \left(-\rho^{\varepsilon}\right)-\theta\right]=-(1-\alpha) \beta(0)<0 \text {,and }
\end{aligned}
$$

$$
\begin{aligned}
\lim _{x \rightarrow \infty} h(x)= & \lim _{x \rightarrow \infty}(1-\alpha) \gamma e^{-(x+\rho)^{\varepsilon}}+\lim _{x \rightarrow \infty} \gamma e^{-(x+\rho)^{\varepsilon}} \varepsilon x(x+\rho)^{\varepsilon-1} \\
& -(1-\alpha) \theta=-(1-\alpha) \theta<0 .
\end{aligned}
$$

So at any critical point $x$, we have $h^{\prime}(x)=0$. Accordingly,

$$
\gamma e^{-(x+\rho)^{\varepsilon}} \varepsilon(x+\rho)^{\varepsilon-1}\left[-\left(1-\alpha+\varepsilon x(x+\rho)^{\varepsilon-1}\right)+1-(1-\varepsilon) \frac{x}{x+\rho}\right]
$$

$=0$.

Hence, $\left(1-\alpha+\varepsilon x(x+\rho)^{\varepsilon-1}\right)=1-(1-\varepsilon)(x / x+\rho)$. Then we have

$h(x)=\gamma e^{-(x+\rho)^{\varepsilon}}\left[1-(1-\varepsilon) \frac{x}{x+\rho}\right]-(1-\alpha) \theta$

at any critical point $x$. It is clear that the expression $\gamma e^{-(y+\rho)^{\varepsilon}}[1-$ $(1-\varepsilon)(y / y+\rho)]$ is maximized at $y=0$. Therefore, we obtain that $h(x) \leq \gamma e^{-\rho^{\varepsilon}}-(1-\alpha) \theta$. Recall that $\left(\gamma e^{-\rho^{\varepsilon}} / 1-\alpha\right)<\theta$, hence $\gamma e^{-\rho^{\varepsilon}}-(1-\alpha) \theta<0$, implying that at any critical point $x, h(x)<0$.
Thus, $h$ is a negative valued function, implying that $\beta(x) f(x)$ is strictly decreasing.

Any discount function $\beta$ under which $\beta(x) f^{\prime}(x)$ turns out to be a strictly decreasing function implies a unique steady state and global convergence. The unique steady state is both locally and globally determinate. Moreover, the model may even possess a continuum of steady states, and it depends on the initial condition as to which one is realized in the long run. Hence, the economy exhibits no tendency toward global convergence; an important departure with respect to the neoclassical growth model.

Example 3. Consider the following discount function that consists of three parts:

$\beta(x)= \begin{cases}m x+n, & x<a ; \\ \frac{1}{f^{\prime}(x)}=\frac{1}{A \alpha} x^{1-\alpha}, & a \leq x \leq b ; \\ \eta-\frac{\gamma}{x}, & b<x .\end{cases}$

where $A>0,1>\alpha>0,(A \alpha / 2-\alpha)^{1 / 1-\alpha}>b>a>0, \quad m=1-\alpha / A \alpha a^{-\alpha}$, $n=(1 / A) a^{1-\alpha}, \eta=(2-\alpha / A \alpha) b^{1-\alpha}$, and $\gamma=(1-\alpha / A \alpha) b^{2-\alpha}$. Lets check the assumptions. $\beta$ is already differentiable in the regions $(0, a),(a$, $b),(b, \infty)$. Some simple algebra for checking the left and right limits of $\beta$ and $\beta^{\prime}$ at the points $a$ and $b$ shows that this specification of $m$, $n, \eta$ and $\gamma$ makes $\beta$ is differentiable at $a$ and $b$. Therefore $\beta$ is differentiable, also implying continuity. $\beta(0)=n>0$, hence $\beta$ ranges into $\mathbb{R}_{++}$. Also, as $m>0, A>0, \alpha \in(0,1)$, and $\gamma>0, \beta$ is strictly increasing. As $\beta$ is strictly increasing, the supremum of which is $\eta$, is strictly less than one by $(A \alpha / 2-\alpha)^{1 / 1-\alpha}>b$. Moreover, the supremum of the derivative of $\beta$ is also bounded which is easy to see by some algebra.

Notice that throughout the whole interval $[a, b], \beta(x) f^{\prime}(x)=1$, therefore we have a continuum of steady states $[a, b]$. As we have proven that any equilibrium with externality with initial capital $x_{0} \in[a, b]$ will be constant over time, the initial level of capital stock lower than $a$ will lead to an increasingly monotonic convergence towards $a$ whereas the initial the initial level of capital stock higher than $b$ will result in decreasingly monotonic convergence towards $b$.

Remark 2. Existence of the critical value $x_{c}$ and continuum of equilibria with strictly concave production function are peculiar to our model of endogenous time preference.

\section{Conclusion}

In this paper, we present the dynamic implications of endogenous time preference depending on the stock of wealth in a one sector growth model. We prove without any assumption on the curvatures of the production and the discount functions that optimal policy is single valued and differentiable almost everywhere in the planner's problem and the optimal paths are monotonic. We consider the optimal path dynamics in the long run and show that our model can exhibit global convergence even under a convex-concave technology and multiplicity of optimal steady states even under a convex technology. Indeed, we show that there exists a critical stock of capital below which the optimal program leads only to forever diminishing capital and gradually converging to development trap and above which the economy converges to the high steady state. We also show that if it so happens that the initial level of capital stock equals the critical stock, which is not an optimal or nonoptimal steady state, an indeterminacy emerges. Moreover, the multipliers system associated with an optimal path is proven to be the supporting price system of a competitive equilibrium under externality and detailed results 
concerning the properties of optimal (equilibrium) paths are provided.

Extensions to our model are obviously possible and include the considerations of uncertainty, heterogenous agents and strategic interactions. These are in our research agenda.

\section{References}

Amir, R., Mirman, L.J., Perkins, W.R., 1991. One-sector nonclassical optimal growth optimality conditions and comparative dynamics. International Economic Review 32 (3), 625-644.

Askenazy, P., Le Van, C., 1999. A model of optimal growth strategy. Journal of Economic Theory 85 (1), 24-51.

Askri, K., Le Van, C., 1998. Differentiability of the value function of nonclassical optimal growth models. Journal of Optimization Theory and Applications 97, 591-604.

Araujo, 1991. The once but not twice differentiability of the policy function. Econometrica 59 (5), 1383-1393.

Azariadis, C., Stachurski, J., 2005. Poverty Traps. In: Aghion, P., Durlauf, S. (Eds.), In: Handbook of Economic Growth, vol. 1. Elsevier, Amsterdam, pp. 295-384.

Barro, R.J., 1997. Determinants of Economic Growth. MIT Press, Cambridge, MA

Barro, R.J., Sala-i-Martin, X., 1991. Convergence across states and regions. Brookings Papers on Economic Activity 1, 107-182.

Beals, R., Koopmans, T.C., 1969. Maximizing stationary utility in a constant technology. SIAM Journal of Applied Mathematics 17 (5), 1001-1015.

Becker, G.S., Mulligan, C.B., 1997. The endogenous determination of time preference. The Quarterly Journal of Economics 112 (3), 729-758.

Benhabib, J., Farmer, R.E.A., 1996. Indeterminacy and sector specific externalities. Journal of Monetary Economics 37, 21-443.

Benhabib, J., Perli, R., 1994. Uniqueness and indeterminacy: transitional dynamics with multiple equilibria. Journal of Economic Theory 63, 113-142.

Benveniste, L.M., Scheinkman, J.A., 1979. On the differentiability of the value function in dynamic models of economies. Econometrica 47, 727-732.

Clark, C.W., 1971. Economically optimal policies for the utilization of biologically renewable resources. Mathematical Biosciences 17, 245-268.

Das, M., 2003. Optimal growth with decreasing marginal impatience. Journal of Economic Dynamics and Control 27, 1881-1898.

Dechert, W.D., Nishimura, K., 1983. A complete characterization of optimal growth paths in an aggregated model with non-concave production function. Journal of Economic Theory 31, 332-354.

Duran, J., Le Van, C., 2003. Simple proof of existence of equilibrium in a one-sector growth model with bounded or unbounded returns from below. Macroeconomic Dynamics 7, 317-332.
Epstein, L., 1987. A simple dynamic general equilibrium model. Journal of Economic Theory 41, 68-95.

Frederick, S.G., Loewenstein, G., O'Donoghue, T., 2002. Time discounting and time preference: a critical review. Journal of Economic Literature 40, 351-401.

Hamada, K., Takeda, Y., 2009. On the Role of the Rate of Time Preference in Macroeconomics: A Survey. In: International Trade and Economic Dynamics: Essays in Memory of Koji Shimomura ,. Springer, Berlin/Heidelberg, pp. 393-420.

Harrison, G.W., Lau, M.I., Williams, M.B., 2002. Estimating individual discount rates in Denmark: a field experiment. American Economic Review 92, 1606-1617.

Ikeda, S., Ohtake, F., Tsutsui, Y., 2005. Time discount rates: an analysis based on economic experiments and questionnaire surveys. Institute of Social and Economic Research Discussion Paper No. 638, Osaka University.

Iwai, K., 1972. Optimal economic growth and stationary ordinal utility-a fisherian approach. Journal of Economic Theory 5, 121-151.

Kurz, M., 1968. Optimal economic growth and wealth effects. International Economic Review 9, 348-357.

Lawrance, E.C., 1991. Poverty and the rate of time preference: evidence from panel data. Journal of Political Economy 99, 54-75.

Le Van, C., Dana, R.A., 2003. Dynamic Programming in Economics. Kluwer Academic Publishers.

Lucas, R.E., Stokey, N., 1984. Optimal growth with many consumers. Journal of Economic Theory 32, 139-171.

Majumdar, M., Mitra, T., 1982. Intertemporal allocation with a non-convex technology: the aggregative framework. Journal of Economic Theory 27, 101-136.

Mantel, R.R., 1998. Optimal economic growth with recursive preferences: decreasing rate of time preferences. Estudios de Economia 25, 161-178.

Mitra, T., Ray, D., 1984. Dynamic optimization on non-convex feasible set: some general results for non-smooth technologies. Zeitschrift fur Nationaokonomie $44,151-175$.

Nishimura, K., Venditti, A., 2006. Indeterminacy in discrete-time infinite-horizon models. In: Dana, R.A., Le Van, C., Mitra, K., Nishimura, K. (Eds.), Handbook on Economic Growth: Vol. 1: The Discrete Time Horizon. Kluwer.

Obstfeld, M., 1990. Intertemporal dependence, impatience and dynamics. Journal of Monetary Economics 26, 45-75.

Quah, D.T., 1996. Convergence empirics across economies with (some) capital mobility. Journal of Economic Growth 1, 95-124.

Samwick, A., 1998. Discount rate homogeneity and social security reform. Journal of Development Economics 57, 117-146.

Skiba, A.K., 1978. Optimal growth with a convex-concave production function. Econometrica 46, 527-539.

Stern, M.L., 2006. Endogenous time preference and optimal growth. Economic Theory $29,49-70$.

Stokey, N.L., Lucas, R., Prescott, E., 1989. Recursive Methods in Economic Dynamics. Harvard University Press.

Uzawa, H., 1968. Time preference, the consumption function, and optimum asset holdings. In: Wolfe, J. (Ed.), Value, Capital and Growth, Chicago. Chicago University Press. 